\title{
Cerebellar Hemorrhage Secondary to Cerebellopontine Angle Metastasis From Thyroid Papillary Carcinoma -Case Report-
}

\author{
Toshihide TANAKA, ${ }^{1}$ Naoki KATO,${ }^{1}$ Ken AOKI, ${ }^{1}$ Aya NAKAMURA, ${ }^{1}$ \\ Mitsuyoshi WATANABE, ${ }^{1}$ Takao ARAI, ${ }^{1}$ Yuzuru HASEGAWA, ${ }^{1}$ \\ Kensuke AOKI, ${ }^{2}$ Kazuhisa YAMAMOTO, ${ }^{2}$ and Toshiaki $\mathrm{ABE}^{3}$ \\ Departments of ${ }^{1}$ Neurosurgery and ${ }^{2}$ Otorhinolaryngology, \\ Jikei University School of Medicine Kashiwa Hospital, Kashiwa, Chiba; \\ ${ }^{3}$ Department of Neurosurgery, Jikei University School of Medicine, Tokyo
}

\begin{abstract}
A 63-year-old woman presented with a metastatic focus of papillary thyroid carcinoma in the cerebellopontine angle manifesting as lateral gazing nystagmus and slurred speech. Computed tomography demonstrated massive hemorrhage in the left cerebellar hemisphere. She was treated conservatively. Her symptoms resolved completely, but she experienced progressive deterioration in auditory acuity and ataxia over the next 6 months. Magnetic resonance imaging with gadolinium demonstrated an enhanced mass in the left cerebellopontine angle, and she was scheduled for elective resection of the tumor. Left retrosigmoid craniotomy was performed, and the tumor was subtotally removed except for a small amount at the junction of the trigeminal nerve and the pons. Histological examination confirmed a diagnosis of metastatic papillary thyroid carcinoma. The patient then underwent adjuvant gamma knife radiosurgery. Her clinical course was unremarkable, and her hypoacusis and ataxia resolved completely. Postoperative gallium scintigraphy revealed no residual tumor and no other systemic metastases. Hemorrhagic cerebellar metastasis from papillary thyroid carcinoma is extremely rare, but early recognition of metastatic cerebellar tumor should prompt immediate treatment to avoid the development of hearing loss, ataxia, and tonsillar herniation.
\end{abstract}

Key words: cerebellar hemorrhage, cerebellopontine angle metastasis, papillary thyroid carcinoma, surgery

\section{Introduction}

Thyroid carcinoma occurs in 1 in 25,000 people. ${ }^{18)}$ Papillary carcinoma is the most common type of well-differentiated carcinoma of the thyroid, accounting for $81.2 \%$ of all thyroid malignancies. ${ }^{4,13)}$ Papillary carcinoma is typically indolent, and the overall prognosis is usually excellent. Survival rates for non-metastatic lesions are $97.8 \%$ at 5 years and $94.9 \%$ at 10 years. ${ }^{13,14)}$ Metastasis of papillary thyroid carcinoma to regional lymph nodes, including the cervical and upper mediastinal nodes, ${ }^{4)}$ occurs in $9 \%$ to $10 \%$ of patients. ${ }^{17)}$ However, brain metastasis from thyroid carcinoma is rare, occurring in only $0.1 \%$ to $5 \%$ of cases in reported series. ${ }^{1,2,6,7,12,16)}$ Metastasis of thyroid carcinoma to the cerebellopontine angle is even rarer, with only three such reports. ${ }^{1,2,5,6)}$ Hemorrhagic brain metastases can occur in the context of melanoma, choriocarcinoma, renal cell carcinoma, and bronchogenic carcinoma. By contrast, solitary hemorrhagic cerebellar metastasis from papillary

Received October 4, 2011;

Accepted January 24, 2012 thyroid carcinoma is extremely rare.$^{10-12,15)}$ We describe a case of a patient with metastasis of papillary thyroid carcinoma to the cerebellopontine angle associated with intratumoral hemorrhage, which was initially misdiagnosed with simple cerebellar hemorrhage, resulting in delay of treatment.

\section{Case Report}

A 63-year-old woman presented with sudden onset of headache and dizziness in October 2010 and was referred to our hospital. She had previously undergone total resection of papillary carcinoma of the thyroid in July 2005, and the postoperative course was uneventful without local recurrence or distant metastases. Iodine-131 ( $\left.{ }^{131} \mathrm{I}\right)$ scintigraphy detected no accumulation, so no adjuvant therapies including radioactive iodine treatment were given. She was noted to have bilateral horizontal gaze nystagmus and slurred speech on examination. Computed tomography (CT) demonstrated massive hemorrhage in the left cerebellar hemisphere, which was not typical of cerebellar 
hemorrhage caused by hypertension (Fig. 1A). She had no history of hypertension.

Magnetic resonance (MR) imaging showed no intense enhancement with gadolinium. Cerebral angiography showed no abnormal vessels or aneurysms (data not shown). Initially we suspected cavernous angioma. After conservative follow-up for 3 weeks, her symptoms resolved entirely. CT showed that the hematoma had been completely absorbed (Fig. 1B). However, over the next 6 months, she experienced gradual deterioration in auditory acuity and ataxia. CT in April 2011 revealed hematoma in the cerebellopontine angle (Fig. 1C), and MR imaging with
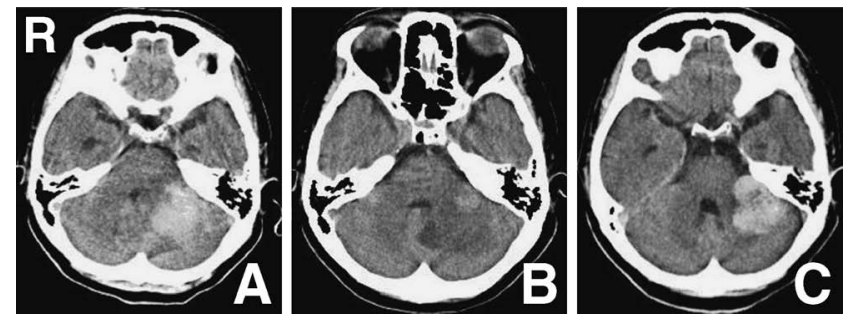

Fig. 1 Initial computed tomography (CT) scan in November 2010 (A) showing cerebellar hemorrhage in the left cerebellar hemisphere, and follow-up CT scan 2 weeks after onset (B) revealing absorption of the hematoma. The patient experienced progressive deterioration in auditory acuity. CT scan taken in April 2011 (C) demonstrating massive extraaxial hematoma in the left cerebellopontine angle.
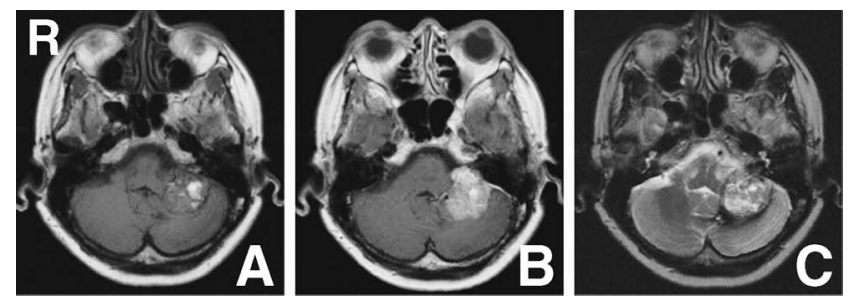

Fig. 2 Preoperative $T_{1}$-weighted magnetic resonance (MR) image without (A) and with gadolinium (B) showing an isointense and well-enhanced mass lesion, and $T_{2}$-weighted MR image (C) showing a hyperintense tumor in the left cerebellopontine angle. gadolinium demonstrated a $2.1 \times 3.0 \mathrm{~cm}$ enhanced mass in the left cerebellopontine angle (Fig. 2). She was noted to have left hypoacusis, and left Bruns nystagmus with diplopia in May 2011. Audiography showed decreased acuity (Fig. 3A, B). The patient was scheduled for elective resection of the tumor. Over the next week, she developed left hearing loss (Fig. 3B) and inability to walk for which she was admitted to the hospital.

Left retrosigmoid craniotomy was performed. The hypervascular tumor was located anterior to and was compressing the cerebellum posteriorly. The tumor extended medially to the pons and displaced the trigeminal nerve superiorly. The acoustic-facial nerve bundle and lower cranial nerves were identified and dissected free from the tumor. The tumor was also dissected free from the dura in the skull base. Facial nerve monitoring was performed during the operation, and the tumor was dissected free without injury to the facial and acoustic nerves. The border of the tumor was clearly detected, but was firmly attached to the cerebellum and the trigeminal nerve. To preserve the nerve, a small amount of the tumor was left in place at the junction of the trigeminal nerve and the pons. Histological findings of a rapid frozen section were consistent with a diagnosis of metastatic papillary tumor. Postoperatively, the patient's auditory acuity improved (Fig. 3C), and she remained free from neurological deficits. Her postoperative course was uneventful, and she was discharged 9 days after the operation.
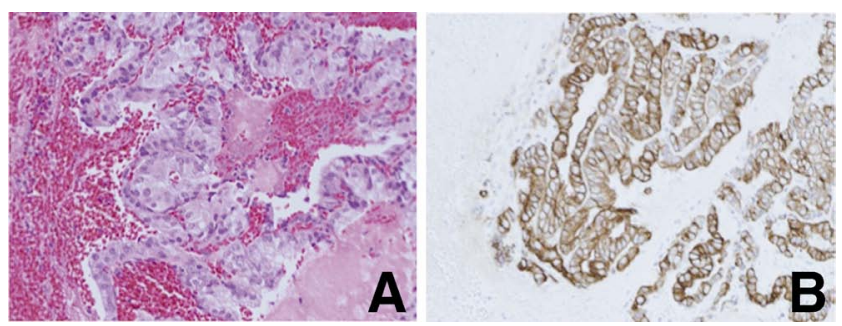

Fig. 4 A: Photomicrograph of the papillary adenocarcinoma showing papillae with columnar cells with abundant cytoplasm, and tumor cells were involved in the massive hemorrhage. Hematoxylin and eosin stain, original magnification $\times 200$. B: Immunohistochemical staining with CK7 revealing strong positive staining in tumor cells. Original magnification $\times 200$.
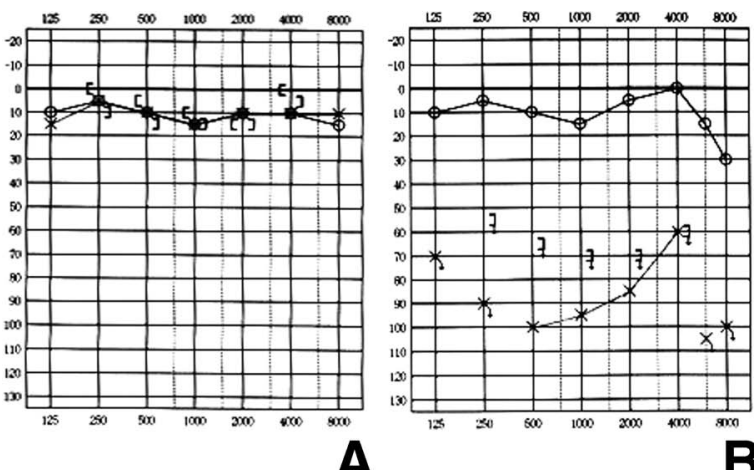

A

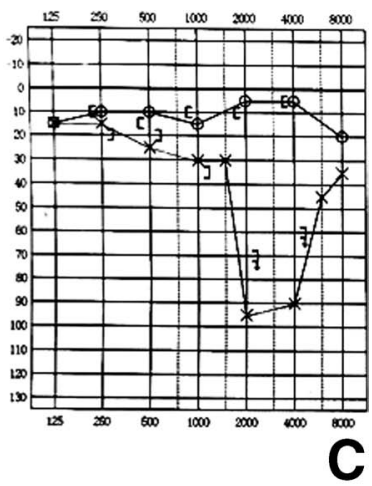

Fig. 3 Audiograms prior to surgery. Bilateral auditory acuity was within normal limits in April (A), but rapid deterioration of hearing loss was recognized in May (B). Surgery was scheduled following the second audiogram. Postoperative hearing acuity improved, especially in the range between 250 and $1000 \mathrm{~Hz}$ (C). 
Histological examination revealed papilla with a fibrovascular core lined by tall cells with abundant cytoplasm (Fig. 4A). Immunohistochemistry was positive for CK7 and thyroglobulin, and was negative for CK20 (Fig. 4B). These findings were compatible with a diagnosis of metastatic tumor from thyroid papillary carcinoma.

Postoperative MR imaging one week after the operation revealed subtotal removal of the tumor in the cerebellopontine angle except for a small amount adjacent to the pons (Fig. 5). Gallium scintigraphy showed no signs of other metastatic foci. The patient underwent adjuvant gamma knife radiosurgery with a dose of 15 Gy at the margin. Her hypoacusis and ataxia had completely resolved at follow-up examination. Her clinical course was uneventful 23 months after operation.

\section{Discussion}

Papillary thyroid carcinoma usually metastasizes to local regional lymph nodes, most commonly the cervical and upper mediastinal lymph nodes, and distant metastasis is less common. ${ }^{13,14)}$ The lung is the most common site of distant metastases (77\%) followed by the bones $(20 \%)^{8,14)}$ Brain metastases are extremely rare, as the incidence of cerebral metastases of papillary thyroid carcinoma is approximately $0.1 \%$ to $5 \% .^{5,9,14,18)}$ Cerebral metastases usually present in association with previously treated thyroid tumors or with disseminated metastases. The average interval between the diagnosis of primary cancer and brain metastasis is 4 years (1-35 years), ${ }^{1,7)}$ but brain metastasis is
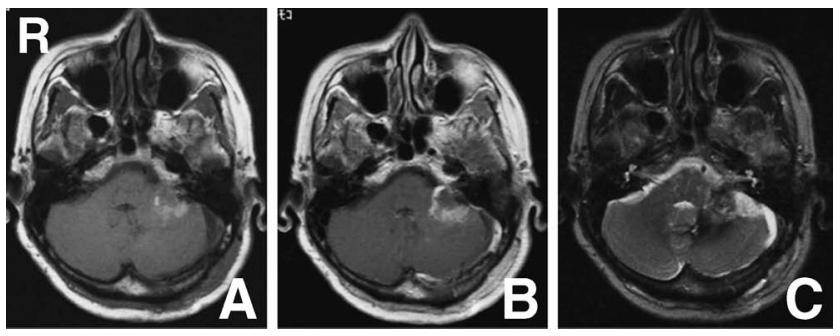

Fig. 5 Postoperative $T_{1}$-weighted magnetic resonance (MR) image without (A) and with gadolinium (B), and $T_{2}$-weighted MR image (C), showing the tumor was completely removed except for some residual tumor adjacent to the pons. rarely discovered as the first metastasis before the primary lesion. ${ }^{17)}$ Presence of brain metastases is an overall negative indicator, and overall survival of patients with brain metastases is 3 to 14 months if the tumor was treated by radiotherapy. ${ }^{1,6)}$ In contrast, prognosis is longer if the tumor was surgically resected with or without adjuvant therapy. Only three cases of papillary thyroid carcinoma metastases located in the cerebellopontine angle have been reported (Table 1). ${ }^{1,5,6)}$ Prognosis of cerebellopontine angle tumors seems to be favorable compared to the other sites.

The present patient initially presented with cerebellar hemorrhage, and it was not recognized that the hematoma resulted from underlying metastasis of thyroid cancer. Such hemorrhagic cerebellopontine angle metastasis from papillary thyroid carcinoma is apparently unique (Table 1). There is no clearly defined treatment protocol for patients with intracranial metastatic tumors from papillary thyroid carcinoma, and therapy must be individually modified for each patient. Several treatment modalities have been used, including surgical resection, external beam radiation, gamma knife radiosurgery, and radioiodine therapy. Papillary thyroid carcinoma is known to be sensitive to internal beam radiation therapy. However, ${ }^{131}$ I may or may not accumulate in the metastatic tumor tissue of papillary thyroid cancer, as previously reported.6) Patients with brain metastases from thyroid cancer treated by stereotactic radiosurgery had an overall median survival time of 20.8 months, ${ }^{3)}$ which is more favorable than any other study, ${ }^{1,6)}$ suggesting that stereotactic radiosurgery can provide a favorable prognosis with an expected median survival greater than 3 years. ${ }^{3}$ Therefore, we selected gamma knife as an adjuvant therapy in the present case. Surgery is the treatment of choice for lifethreatening complications, such as increased intracranial pressure, cerebral edema, tonsillar herniation, or intracranial hemorrhage. Surgery is occasionally scheduled on semi-urgent basis due to deteriorating neurological function, especially in patients with metastatic tumors in the posterior fossa.

In the present case, intratumoral hemorrhage resulted in progressive dizziness and hearing loss, so surgery was necessary to confirm the diagnosis and to reduce the aggravating increase in intracranial pressure due to tumor hemorrhage. Initial CT demonstrated hemorrhage in the

Table 1 Summary of cases of cerebellopontine angle metastasis from papillary thyroid carcinoma

\begin{tabular}{|c|c|c|c|c|c|c|c|}
\hline Author (Year) & $\begin{array}{l}\text { Age (yrs)/ } \\
\text { Sex }\end{array}$ & Side & $\begin{array}{l}\text { Intratumoral } \\
\text { hemorrhage }\end{array}$ & $\begin{array}{l}\text { Single/ } \\
\text { multiple }\end{array}$ & $\begin{array}{l}\text { Interval until } \\
\text { brain metastases } \\
\text { (yrs) }\end{array}$ & Treatment & Outcome \\
\hline $\begin{array}{l}\text { Carcangiu et al. } \\
(1985)^{5)}\end{array}$ & $50 / \mathrm{F}$ & $\mathrm{rt}$ & - & single & n.d. & radioactive iodine & $\begin{array}{l}\text { no recurrence in } \\
8 \mathrm{yrs}\end{array}$ \\
\hline $\begin{array}{l}\text { Cha et al. } \\
\qquad(2000)^{6)}\end{array}$ & $78 / \mathrm{F}$ & lt & - & single & 3 & surgery/gamma knife & $\begin{array}{l}\text { no recurrence in } \\
36 \mathrm{mos}\end{array}$ \\
\hline $\begin{array}{l}\text { Aguiar et al. } \\
(2001)^{1)}\end{array}$ & $33 / \mathrm{F}$ & $\mathrm{rt}$ & - & multiple & 3 & surgery/radiotherapy & $\begin{array}{l}\text { no recurrence in } \\
36 \mathrm{mos}\end{array}$ \\
\hline Present case & $63 / F$ & lt & + & single & 6 & surgery/gamma knife & $\begin{array}{l}\text { no recurrence in } \\
23 \mathrm{mos}\end{array}$ \\
\hline
\end{tabular}

F: female, lt: left, M: male, n.d.: not described, rt: right. 
cerebellar hemisphere. Therefore, cavernous angioma and occult arteriovenous malformation were suspected. However, follow-up CT revealed hematoma in the cerebellopontine cistern instead of the cerebellar hemisphere. In addition, hearing acuity declined over a relatively short period of time. This led to consideration of the possibility of intratumoral hemorrhage caused by acoustic neurinoma or metastasis as alternate diagnoses. Since only histological examination provides confirmation of the diagnosis, we decided on surgical operation as the first choice.

Tumors most frequently associated with hemorrhagic brain metastasis include choriocarcinoma, melanoma, renal cell carcinoma, bronchogenic carcinoma, and hepatocellular carcinoma. Only sporadic cases of hemorrhagic brain metastasis have been reported in association with papillary thyroid carcinoma. ${ }^{10-12,15)}$ The present case demonstrates that metastatic papillary thyroid carcinoma should be included in the differential diagnosis of solitary hemorrhagic tumor in the cerebellum. Early recognition of intracranial tumor should prompt immediate institution of a treatment plan. In general, papillary thyroid carcinoma has a good prognosis, with an overall mortality rate of less than $6.5 \%$. Surgical resection of brain metastases significantly prolongs survival in patients with differentiated thyroid carcinoma.7) However, distant metastases and local tumor infiltration can be fatal, as supported by observations that central nervous system metastases from papillary thyroid carcinoma were associated with high mortality rates. ${ }^{14)}$

Underlying brain metastases should always be considered in patients with intracranial hematoma. Aggressive treatment may improve long-term outcomes for patients presenting with central nervous system metastases, especially for those with cerebellar lesions, since cerebellar tumors can cause life-threatening complications, such as tonsillar herniation. Although there are no definitive guidelines for the management of brain metastasis from thyroid cancer, a surgical approach should be considered prior to ${ }^{131}$ I therapy. If advanced disease or neurological symptoms are present, surgical removal of the metastatic tumor should be considered to prevent life-threatening complications.

\section{Conflicts of Interest Disclosure}

All authors have no conflict of interest. All authors who are members of The Japan Neurosurgical Society (JNS) have registered online Self-reported COI Disclosure Statement Forms through the website for JNS members.

\section{References}

1) Aguiar $\mathrm{PH}$, Agner $\mathrm{C}$, Tavares FR, Yamaguchi N: Unusual brain metastases from papillary thyroid carcinoma: Case report. Neurosurgery 49: 1008-1013, 2001

2) Al-Dhahri SF, Al-Amro AS, Al-Shakwer W, Terkawi AS: Cerebellar mass as a primary presentation of papillary thyroid carcinoma: case report and literature review. Head Neck Oncol 1: 23, 2009
3) Bernad DM, Sperduto PW, Souhami L, Jensen AW, Roberge $\mathrm{D}$ : Stereotactic radiosurgery in the management of brain metastases from primary thyroid cancers. J Neurooncol 98: 249-252, 2010

4) Boone RT, Fan CY, Hanna EY: Well-differentiated carcinoma of the thyroid. Otolaryngol Clin North Am 36: 73-90, 2003

5) Carcangiu ML, Zampi G, Pupi A, Castagnoli A, Rosai J: Papillary carcinoma of the thyroid; a clinicopathologic study of 241 cases treated at the University of Florence, Italy. Cancer 55: 805-828, 1985

6) Cha ST, Jarrahy R, Mathiesen RA, Suh R, Shahinian HK: Cerebellopontine angle metastasis from papillary carcinoma of the thyroid: case report and literature review. Surg Neurol 54: $320-326,2000$

7) Chiu AC, Delpassand ES, Sherman SI: Prognosis and treatment of brain metastases in thyroid carcinoma. J Clin Endocrinol Metab 82: 3637-3642, 1997

8) Dinneen SF, Valmaki MJ, Bergstralh EJ, Gorman CA, Hay ID: Distant metastasis in papillary thyroid carcinoma; 100 cases observed at one institution during 5 decades. J Clin Endocrinol Metab 80: 2040-2045, 1995

9) Hoie J, Sienwig AE, Kullmann G, Lindegaard M: Distant metastasis in papillary thyroid cancer: a review of 91 patients. Cancer 61: 1-6, 1988

10) Isoda $H$, Takahashi $M$, Arai $T$, Ramsey RG, Yokoyama $T$, Mochizuki T, Yamamoto I, Kaneko M: Multiple haemorrhagic brain metastases from papillary thyroid cancer. Neuroradiology 39: 198-202, 1997

11) Lecumberri B, Alvarez-Escola $C$, Martin-Vaquero $P$, Nistal M, Martin V, Riesco-Eizaguirre G, Sosa G, Pallardo LF: Solitary hemorrhagic cerebellar metastasis from occult papillary thyroid microcarcinoma. Thyroid 20: 563-567, 2010

12) Lin CK, Lieu AS, Howng SL: Hemorrhagic cerebellar metastasis from papillary thyroid carcinoma. Kaohsiung $J$ Med Sci 15: 234-238, 1999

13) Mazzaferri EL, Young R, Omertel JE, Kemmeer WT, Page CP: Papillary thyroid carcinoma; the impact of therapy in 576 patients. Medicine 56: 171-196, 1977

14) McConahey WM, Hay ID, Weelner LB, van Heerden JA, Taylor WF: Papillary thyroid cancer treated at Mayo Clinic, 1946 through 1970; initial manifestations, pathologic findings, therapy, and outcome. Mayo Clin Proc 61: 978-996, 1986

15) Pacak K, Sweeney DC, Wartofsky L, Mark AS, Punja U, Azzam CJ, Burman KD: Solitary cerebellar metastasis from papillary thyroid carcinoma: A case report. Thyroid 8: 327-335, 1998

16) Pazaitou-Panayiotou K, Kaprara A, Chrisoulidou A, Boudina M, Georgiou E, Patakiouta F, Drimonitis A, Vainas I: Cerebellar metastasis as first metastasis from papillary thyroid carcinoma. Endocr J 52: 653-657, 2005

17) Tuttle RM, Leboeuf R, Martorella AJ: Papillary thyroid cancer: monitoring and therapy. Endocrinol Metab Clin North Am 36: 753-778, 2007

18) Venkatesh S, Leavens ME, Samaan NA: Brain metastases in patients with well-differentiated thyroid carcinoma; study of 11 cases. Eur J Surg Oncol 16: 448-450, 1990

Address reprint requests to: Toshihide Tanaka, MD, PhD, Department of Neurosurgery, Jikei University School of Medicine Kashiwa Hospital, 163-1 Kashiwa-shita, Kashiwa, Chiba 277-8567, Japan.

e-mail: ttanaka@jikei.ac.jp 